PROCEEDINGS OF THE

AMERICAN MATHEMATICAL SOCIETY

Volume 131, Number 11, Pages 3583-3588

S 0002-9939(03)07118-1

Article electronically published on June 18, 2003

\title{
NOTE ON THE RIEMANN-HURWITZ TYPE FORMULA FOR MULTIPLICATIVE SEQUENCES
}

\author{
TAKESHI IZAWA
}

(Communicated by Mohan Ramachandran)

\begin{abstract}
We give a formula of the Riemann-Hurwitz type for classes defined by multiplicative sequences as corollaries of the Chern number formula for ramified coverings.
\end{abstract}

1.

1.1. In the classical results on Riemann surfaces, we have the Riemann-Hurwitz formula which relates the topological Euler numbers of the covering space and base space. The difference of the Euler numbers can be expressed by local residues of ramification points. In the higher-dimensional case, we can see that the difference of the Euler numbers can be expressed by Euler numbers of degeneration loci ([Y]). In the simplest case that a covering map $f: X \longrightarrow Y$ between $n$-dimensional compact complex manifolds with covering multiplicity $\mu$ has the non-singular branch locus $B$, the above formula is

$$
\chi(Y)-\mu \cdot \chi(X)=-\chi(B) .
$$

In such cases, we can also consider the genera of $B$ determined by the multiplicative sequence $K_{j}\left(c_{1}, \cdots, c_{j}\right)$ of Chern classes. Thus we expect that the difference

$$
K_{n}\left(c_{1}(X), \cdots, c_{n}(X)\right)-\mu K_{n}\left(c_{1}(Y), \cdots, c_{n}(Y)\right)
$$

can be explessed by datas of $\left\{K_{j}\right\}_{1 \leq j \leq n-1}$ of $B$. But there are no results for this type of formulas.

In this note, we show that this type of formula follows immediately from the definition of the multiplicative sequence and the Chern number formula for ramified coverings $(\underline{\mathrm{Iz}}])$.

1.2. First we recall the definition of multiplicative sequence. (For more detail, see [Hz1].) Let $A$ be a commutative algebra with identity and consider $A\left[p_{1}, p_{2}, \cdots\right]$, the ring of polynomials in the $p_{i}$. Let $\left\{K_{j}\right\}$ be a sequence of polynomials in the

Received by the editors April 6, 2001.

2000 Mathematics Subject Classification. Primary 57R20; Secondary 32J25.

Key words and phrases. Ramified covering, Riemann-Hurwitz formula, multiplicative sequence.

This article was partially supported by the Japan Society for the Promotion of Science.

(C)2003 American Mathematical Society 
indeterminates $p_{i}$ and write

$$
K\left(\sum_{j=0}^{\infty} p_{j} z^{j}\right):=\sum_{j=0}^{\infty} K_{j}\left(p_{1}, \cdot s, p_{j}\right) z^{j} .
$$

The sequence $K=\left\{K_{j}\right\}$ is called a multiplicative sequence if every identity of the form

$$
\sum_{j=0}^{\infty} p_{j} z^{j}=\left(\sum_{j=0}^{\infty} p_{j}^{\prime} z^{j}\right)\left(\sum_{j=0}^{\infty} p_{j}^{\prime \prime} z^{j}\right)
$$

implies an identity

$$
K\left(\sum_{j=0}^{\infty} p_{j} z^{j}\right)=K\left(\sum_{j=0}^{\infty} p_{j}^{\prime} z^{j}\right) K\left(\sum_{j=0}^{\infty} p_{j}^{\prime \prime} z^{j}\right) .
$$

Now let $X$ be a $n$-dimensional compact complex manifold and $c_{i}(E) \in H^{2 i}(X, \mathbf{Z})$ Chern classes of a holomorphic tangent bundle $E$ over $X$. The (total) $K$-class of $E$ is defined by

$$
K(E)=\sum_{j=0}^{\infty} K_{j}\left(c_{1}(E) \cdots c_{j}(E)\right)
$$

where $\left\{K_{j}\left(c_{1} \cdots c_{j}\right)\right\}$ is a multiplicative sequence. Then for vector bundles $E$ and $F$, it follows from the definition of multiplicative sequence that

$$
K(E \oplus F)=K(E) K(F) .
$$

1.3. Next we recall the Chern number formula for ramified coverings. For a ramified covering with good ramification between $n$-dimensional compact complex manifolds, we have the following formula ([Iz]).

Theorem 1.1 (Chern number formula). Let $f: Y \longrightarrow X$ be a ramified covering with covering multiplicity $\mu$ between compact complex manifolds of dimension $n$, $R_{f}=\sum_{i} r_{i} R_{i}$ the ramification divisor of $f$, and $B_{f}=\sum_{i} b_{i} B_{i}$ the branch locus of $f$. We set $f^{*} B_{i}=\sum_{t} r_{i_{t}} R_{i_{t}}$ where $n_{i_{t}}$ denotes the mapping degree of the induced map $\left.f\right|_{R_{i_{t}}}: R_{i_{t}} \rightarrow B_{i}$ with $b_{i}=\sum_{t} n_{i_{t}} r_{i_{t}}$. We assume that the ramification divisor and the irreducible components $B_{i}$ of the branch locus $B_{f}$ are all non-singular, and suppose that $n=\sum_{i+1}^{n} i \cdot N_{i}$. Then:

$$
\begin{aligned}
& c_{1}^{N_{1}}, \cdots, c_{n}^{N_{n}}(Y)-\mu \cdot c_{1}^{N_{1}}, \cdots, c_{n}^{N_{n}}(X) \\
& =\sum_{i} \sum_{\alpha=0}^{n-1}\left(\sum_{t} \frac{n_{i_{t}}\left(1-\left(r_{i_{t}}+1\right)^{\alpha+1}\right)}{\left(r_{i_{t}}+1\right)^{\alpha}}\right) P_{\alpha}\left(c_{1}\left(B_{i}\right), \cdots, c_{n-1}\left(B_{i}\right)\right) \cdot c_{1}\left(L_{B_{i}}\right)^{\alpha} \frown\left[B_{i}\right]
\end{aligned}
$$

where we set

$$
\begin{aligned}
H_{\xi}^{\left(N_{1} \cdots N_{n}\right)}(l) & =l^{-1}\left(\prod_{i=1}^{n}\left(c_{i}(\xi)+c_{i-1}(\xi) \cdot l\right)^{N_{i}}-c_{1}^{N_{1}} \cdots c_{n}^{N_{n}}(\xi)\right) \\
& =\sum_{\alpha=0}^{n-1} P_{\alpha}\left(c_{1}, \cdots, c_{n-1}\right) l^{\alpha} .
\end{aligned}
$$

Let $Q(x)=K(1+X)$ be a characteristic power series of $K$. For the Chern polynomials determined by multiplicative sequences, we can write explicitly the 
coefficients $P_{\alpha}\left(c_{1}\left(B_{i}\right) \cdots c_{n-1}\left(B_{i}\right)\right)$ in the above formula as follows. By applying the formula for $K_{n}$,

$$
K_{n}\left(c_{1}(X) \cdots c_{n}(X)\right)-\mu K_{n}\left(c_{1}(Y) \cdots c_{n}(Y)\right)
$$

is expressed by the form

$$
l^{-1}\left\{K_{n}\left(c_{1}+l, c_{2}+c_{1} l, \cdots, c_{n}+c_{n-1} l\right)-K_{n}\left(c_{1}, \cdots, c_{n}\right)\right\},
$$

which is

$$
c_{1}\left(L_{B}\right)^{-1}\left[K\left(T B \oplus L_{B}\right)-K(T B)\right]_{n} .
$$

Thus we see that

$$
\begin{aligned}
{\left[K\left(T B \oplus L_{B}\right)-K(T B)\right]_{n} } & =\left[K(T B) K\left(L_{B}\right)-K(T B)\right]_{n} \\
& =\sum_{j=0}^{n-1} K_{j}\left(c_{1}, \cdots, c_{j}\right) K_{n-j}(l, 0, \cdots, 0) .
\end{aligned}
$$

In the above we denote by [ $]_{n}$ the term of degree $n$. For the characteristic power series of $K$, we set

$$
Q(x):=K(1+x)=1+\sum_{j=1}^{\infty} A_{j} x^{j} .
$$

Then we set $l=c_{1}\left(L_{B}\right)$ and we have

$$
K_{j}\left(c_{1}\left(L_{B}\right), 0, \cdots, 0\right)=A_{j} c_{1}\left(L_{B}\right)^{j} .
$$

Therefore we obtain the following formula.

Theorem 1.2 (Riemann-Hurwitz formula for $K$-genus). The assumption on settings is the same as above. Let $K$ be a multiplicative sequence and $Q(x):=$ $K(1+x)=1+\sum_{j=1}^{\infty} A_{j} x^{j}$ the characteristic power series of $K$. Then we have

$$
\begin{aligned}
& K_{n}\left(c_{1}(X) \cdots c_{n}(X)\right)-\mu K_{n}\left(c_{1}(Y) \cdots c_{n}(Y)\right) \\
& =\sum_{i} \sum_{\alpha=0}^{n-1} A_{\alpha}\left(\sum_{t} \frac{n_{i_{t}}\left(1-\left(r_{i_{t}}+1\right)^{\alpha+1}\right)}{\left(r_{i_{t}}+1\right)^{\alpha}}\right) K_{n-\alpha-1}\left(c_{1}\left(B_{i}\right), \cdots, c_{n-1}\left(B_{i}\right)\right) \\
& \cdot c_{1}\left(L_{B_{i}}\right)^{\alpha} \frown\left[B_{i}\right] .
\end{aligned}
$$

\section{EXAMPLES}

2.1. Euler number. The result for the top Chern class implies the generalized Riemann-Hurwitz formula

$$
\chi(X)-\mu \cdot \chi(Y)=-\sum_{i} b_{i} \cdot \chi\left(B_{i}\right),
$$

which is a special case of the formula proved by Y. Yomdin [Y]. 
2.2. Signature of surface. From the fact that the signature of the surface is expressed by $L_{1}$, we see that the residue of $L_{1}$ consists only of the self-intersection number of $B$. Thus we have the formula for signature:

Theorem 2.1 (The formula for signature for ramified coverings). Let $f: X \longrightarrow Y$ be a ramified covering between compact complex analytic surfaces with covering multiplicity $\mu, R_{f}=\sum_{i} r_{i} R_{i}$ the ramification divisor of $f$, and $B_{f}=\sum_{i} b_{i} B_{i}$ the branch locus of $f$. We assume that the ramification divisor and irreducible components $B_{i}$ of the branch locus $B_{f}$ are all non-singular. Then

$$
\begin{aligned}
\operatorname{Sign}(X)-\mu \cdot \operatorname{Sign}(Y) & =\frac{1}{3}\left(p_{1}(X)-\mu \cdot p_{1}(Y)\right) \\
& =-\sum_{i} \frac{n_{i_{t}} r_{i_{t}}\left(r_{i_{t}}+2\right)}{3\left(r_{i_{t}}+1\right)} \int_{B_{i}} c_{1}^{2}\left(B_{i}\right) \\
& =-\sum_{i} \frac{n_{i_{t}} r_{i_{t}}\left(r_{i_{t}}+2\right)}{3\left(r_{i_{t}}+1\right)} B_{i} \cdot B_{i} .
\end{aligned}
$$

Originally, the formula for signature for cyclic coverings is formulated for 4manifolds as follows.

Theorem 2.2 (Hirzebruch [Hz2]). Let $X$ be a compact oriented differentiable manifold of dimension 4 without boundary on which the cyclic groups $G_{n}$ of order $n$ act by orientation-preserving diffeomorphisms. Suppose that $Y$ is a differential submanifold of $X$, not necessarily connected, and has codimension 2 , and that $G_{n}$ operates freely on $X-Y$. Then

$$
\operatorname{Sign}(X)-n \cdot \operatorname{Sign}\left(X / G_{n}\right)=-\frac{n^{2}-1}{3 n} Y^{\prime} \cdot Y^{\prime}
$$

where $Y^{\prime}$ is the branch locus in $X / G_{n}$.

This is a particular case of the above formula for signature for ramified coverings, the case that $r=b=n-1$.

2.3. Todd genus. The Todd polynomials $T_{j}$ are determined by the characteristic power series

$$
Q(x)=\frac{x}{1-e^{-x}}=1+\frac{1}{2} x+\sum_{k=1}^{\infty}(-1)^{k-1} \frac{B_{k}}{(2 k) !} B_{k} x^{2 k}
$$

where $B_{k}$ are the Bernoulli numbers. As an example, let us compute the formula for $T_{6}$. The first few terms of the above series are:

$$
Q(l x)=1+\frac{l}{2} x+\frac{l^{2}}{12} x^{2}-\frac{l^{4}}{720} x^{4}+\frac{l^{6}}{27240} x^{6}-\cdots,
$$


which implies the following formula:

$$
\begin{aligned}
T_{6}(Y)-\mu \cdot T_{6}(X)= & -\sum_{i} n_{i_{t}} r_{i_{t}} \frac{T_{5}\left(B_{i}\right)}{2} \\
& +\sum_{i} \sum_{t} \frac{n_{i_{t}} r_{i_{t}}\left(1-\left(r_{i_{t}}+1\right)^{2}\right)}{\left(r_{i_{t}}+1\right)} \int_{B_{i}} \frac{T_{4}\left(B_{i}\right) \smile c_{1}\left(N_{B_{i}}\right)}{12} \\
& -\sum_{i} \sum_{t} \frac{n_{i_{t}} r_{i_{t}}\left(1-\left(r_{i_{t}}+1\right)^{4}\right)}{\left(r_{i_{t}}+1\right)^{3}} \int_{B_{i}} \frac{T_{2}\left(B_{i}\right) \smile c_{1}^{3}\left(N_{B_{i}}\right)}{720} \\
& +\sum_{i} \sum_{t} \frac{n_{i_{t}} r_{i_{t}}\left(1-\left(r_{i_{t}}+1\right)^{6}\right)}{\left(r_{i_{t}}+1\right)^{5}} \int_{B_{i}} \frac{c_{1}^{5}\left(N_{B_{i}}\right)}{27240} .
\end{aligned}
$$

2.4. L-genus. The L polynomials $L_{j}$ are determined by the characteristic power series:

$$
Q(x)=\frac{\sqrt{x^{2}}}{\tanh \sqrt{x^{2}}}=1+\sum_{k=1}^{\infty}(-1)^{k-1} \frac{2^{2 k} B_{k}}{(2 k) !} B_{k} x^{2 k} .
$$

We also compute the formula for the 6-dimensional case, that is, for $L_{3}$. The first few terms of the expansion are:

$$
Q(l x)=1+\frac{l^{2}}{3} x^{2}-\frac{l^{4}}{45} x^{4}+\frac{2 l^{6}}{945} x^{6}+\cdots
$$

Thus we have

$$
\begin{aligned}
L_{3}(X)-\mu \cdot L_{3}(Y)=- & \sum_{i} \sum_{t} \frac{n_{i_{t}} r_{i_{t}}\left(1-\left(r_{i_{t}}+1\right)^{2}\right)}{\left(r_{i_{t}}+1\right)} \int_{B_{i}} \frac{L_{2}\left(B_{i}\right)}{3} \smile c_{1}\left(L_{B_{i}}\right) \\
& -\sum_{i} \sum_{t} \frac{n_{i_{t}} r_{i_{t}}\left(1-\left(r_{i_{t}}+1\right)^{4}\right)}{\left(r_{i_{t}}+1\right)^{3}} \int_{B_{i}} \frac{L_{1}\left(B_{i}\right)}{45} \smile c_{1}\left(L_{B_{i}}\right)^{3} \\
& -\sum_{i} \sum_{t} \frac{n_{i_{t}} r_{i_{t}}\left(1-\left(r_{i_{t}}+1\right)^{6}\right)}{\left(r_{i_{t}}+1\right)^{5}} \int_{B_{i}} \frac{2 c_{1}\left(L_{B_{i}}\right)^{5}}{945}
\end{aligned}
$$

\section{REFERENCES}

[Br1] J. P. Brasselet, Une généralisation de la formule de Riemann-Hurwitz, Bull. Soc. Math. France Suppl. Mémoire 38, 1974, 99-106. MR 50:13622

[D] E. H. C. M. Diop, Résidus d'applications holomorphes entre variétés, Hokkaido Math. J. 29, 2000, no. 1, 171-200. MR 2001g:32077

[GH] P. Griffiths and J. Harris, Principles of Algebraic Geometry, John Wiley \& Sons, New York, Chichester, Brisbane, Toronto, 1978. MR 80b:14001

[Hz1] F. Hirzebruch, Topological Methods in Algebraic Geometry, Springer-Verlag, New York, Heidelberg, Berlin, 1966. MR 34:2573

[Hz2] F. Hirzebruch, The signature of ramified coverings, Global Analysis (Papers in honor of K. Kodaira), Univ. Tokyo Press, Tokyo, 1969, pp. 253-265. MR 41:2707

[Iv] B. Iversen, Numerical invariants and multiple planes, Amer. J. Math. 92, 1970, 968-996. $\operatorname{MR}$ 45:5135 
[Iz] T. Izawa, Chern number formula for ramified coverings, J. Math. Soc. Japan, 52, No. 1, 2000, 1-15. MR 2001i:32028

$[\mathrm{Y}] \quad \mathrm{Y}$. Yomdin, The structure of strata $\mu=$ const in a critical set of a complete intersection singularity, Singularities, Part 2 (Arcata, Calif., 1981), Proc. Sympos. Pure Math., Amer. Math. Soc., Providence, R.I., 1983, 663-665. MR 85a:32013

Department of Mathematics, Faculty of Science, Hokkaido University, Sapporo 060, JAPAN

E-mail address: t-izawa@math.sci.hokudai.ac.jp 